\title{
TOOLS TO ESTIMATE THE FIRST PASSAGE TIME TO A CONVEX BARRIER
}

\author{
OLA HAMMARLID, ${ }^{*}$ Stockholm University
}

\begin{abstract}
The first passage time of a random walk to a barrier (constant or concave) is of great importance in many areas, such as insurance, finance, and sequential analysis. Here, we consider a sum of independent, identically distributed random variables and the convex barrier $c b(n / c)$, where $c$ is a scale parameter and $n$ is time. It is shown, using large-deviation techniques, that the limit distribution of the first passage time decays exponentially in $c$. Under a tilt of measure, which changes the drift, four properties are proved: the limit distribution of the overshoot is distributed as an overshoot over a linear barrier; the stopping time is asymptotically normally distributed when it is properly normalized; the overshoot and the asymptotic normal part are asymptotically independent; and the overshoot over a linear barrier is bounded by an exponentially distributed random variable. The determination of the function that multiplies the exponential part is guided by consideration of these properties.
\end{abstract}

Keywords: First passage time; stopping time; large deviation; rate function; sequential analysis; convex barrier

2000 Mathematics Subject Classification: Primary 60F10

Secondary 60G50; 60G40; 62L10

\section{Introduction}

Consider a sum $S_{n}=\sum_{i=1}^{n} X_{i}$ that starts at zero and whose increments are independent and identically distributed, and let $c b(n / c)$ be a distant upper convex barrier. The barrier is three-times continuously differentiable with $b(0)>0$ and $c$ a scale parameter.

Example 1. The barrier $b(s)$ can take one of the following forms: $1, s+1, \mathrm{e}^{s}, \mathrm{e}^{-s}, 1+s^{2}$, etc. We choose a convex barrier for two reasons: this case has not been fully studied and convexity of the barrier will preserve convexity of a rate function of the stopping time (to be introduced later).

Definition 1. The time when the process $S_{n}$ passes or hits $c b(n / c)$ first is denoted by $N_{c}=$ $\inf \left\{n: S_{n} \geq c b(n / c)\right\}$, with $N_{c}=\infty$ if $S_{n}<c b(n / c)$ for all $n$.

The overshoot over the barrier is written

$$
Z=S_{N_{c}}-c b\left(\frac{N_{c}}{c}\right) .
$$

What is the asymptotic distribution of the stopping time when the scale parameter $c$ goes to infinity?

Received 17 October 2003; revision received 20 August 2004.

* Postal address: Department of Mathematics, Stockholm University, 10691 Stockholm, Sweden.

Email address: olah@math.su.se 
Note that the scaling of time $n / c$ is natural since it is the same scale as $S_{n} / c$, the scale of large numbers; see the following example.

Example 2. An investor is obliged by the bank to deposit $K=\$ 10000$ for a spread position $S_{n}=\sum_{i=1}^{n} X_{i}$, where the $X_{i}$ are the net loss or profit each day. The bank pays interest $r$ such that at time $s$, measured in years, the deposit is worth $K \mathrm{e}^{r s}$. If $S_{n}$ is less than or equal to the deposit, the spread position is closed. What is the probability of the bank closing the position and what is the distribution of the stopping time of this event?

Assume that $r=0.0365$ and $s=n / 365$; then $r s=\left(365 / 10^{4}\right) \cdot(n / 365)=n / 10^{4}$. Under these assumptions, it is natural to put $c=10^{4}$ and, thus, $c b(n / c)=c \mathrm{e}^{n / c}$. The probability of the bank closing the position can be approximated by the asymptotic distribution derived in this article (using $-S_{n}$ as the process).

In the early literature on insurance, the probability of ever hitting a constant barrier - a prospect that meant ruin - was studied by Cramér [3] and Lundberg [21]. They found that the probability of ruin decayed exponentially with a constant rate as the distance to the barrier increased.

The probability of ruin (also before a finite time) has been analysed by the use of many different techniques: two-dimensional renewal theory [15]; ladder variables [30]; integral equations [26]; and martingale techniques [8]. More recently, ruin probability has become of interest in finance. For example, Dembo et al. used large-deviation techniques to study credit risk in a loan or bond portfolio [5]. Further references to the insurance literature can be found in the textbooks by Grandell [8] and Rolski et al. [25].

Renewal theory can be used to study the stopping time to a linear barrier; see, for example, [32]. One method of generalization is to make the barrier time dependent and study the first time at which $S_{n} \geq c b(n)$. The barrier $b(n)=n^{\gamma}$, where $0 \leq \gamma<1$, is nondecreasing, concave, and regularly varying at infinity, and has been studied by Gut [9], [10].

The next possible theoretical step to take is to use nonlinear renewal theory, which is fundamental in sequential analysis. In nonlinear renewal theory, the first passage of $W_{n}=$ $S_{n}+\xi_{n}$ to a linear barrier was analysed by Lai and Siegmund [18], [19]. The sequence $\xi_{n}$ is independent of $S_{m}(m>n)$, slowly changing, and uniformly continuous in probability. As an alternative, let $W_{n}$ be a perturbed random walk, which means that $\xi_{n}$ instead satisfies $\lim _{n \rightarrow \infty} \xi_{n} / n=0$ almost surely. Gut [11] studied the first passage time of a perturbed random walk to a barrier that is nondecreasing, concave, and regularly varying at infinity. (Also see the remarks on [11] made by Larsson-Cohn [20].)

As indicated, the barriers are almost always concave or linear in this area of research (see below for exceptions) and three properties are central. The first property is a central limit theorem of the stopping time. The second is that the overshoot over the barrier converges in distribution to the distribution of an overshoot over a linear barrier. The last property is asymptotic independence between the overshoot and the stopping time. For examples of pioneering work on this, see [13], [18], [19], [29], and [31]. For more references and background on nonlinear renewal theory and sequential analysis, consult the textbooks by Gut [10], Siegmund [28], and Woodroofe [32] for examples.

The first passage density of a Brownian motion, with and without drift to a curved barrier, has been approximated by different schemes; see, for example, [6], [7], [16], and [17]. In the case of diffusions, Roberts and Shortland [24] studied approximations of the stopping time to curved barriers using enveloping curves. The sum $S_{n}$ could, with the right scaling, be approximated by diffusion. This, however, is not the aim here. 
The contribution of this article has several aspects. In the literature on insurance and sequential analysis, the first passage to a convex barrier has not been studied extensively. However, Martin-Löf [22] studied the asymptotic distribution of $\mathrm{P}\left(N_{c} \leq c t\right)$, using Wald's identity and large-deviation techniques. He restricted his analysis to the case $t \geq T$, where $T$ is the most probable value for $N_{c}$. We will use a more probabilistic approach and study the properties for both $t \geq T$ and $t<T$ in more depth.

Siegmund [27] embedded the distribution of $X_{i}$ in an exponential family in order to find the asymptotic distribution of $\mathrm{P}\left(N_{c}<c b(n / c) \mid S_{c}=c \mu_{0}\right)$, where $\mu_{0}<b(1)$. This can be 'translated into knowledge about $\mathrm{P}\left(N_{c}<c b(n / c)\right)$ by integrating out $\mu_{0}$, although a rigorous justification of this approach leads to questions of uniformity in $\mu_{0}$, which may involve additional technical difficulties' [27]. The method used is claimed to work for a large set of barriers, but only the constant barrier and $b(s)=$ const $\cdot s^{1 / 2}$ are analysed here.

We will use large-deviation techniques to prove that $\lim _{c \rightarrow \infty} c^{-1} \log \left(\mathrm{P}\left(N_{c} \leq c t\right)\right)=$ $-\inf _{s \leq t} R(s)$, where $R(s)$ is a rate function of the first passage time. The rate function is equal to a constant $R(T)$ whenever $t \geq T$. The most probable time to pass the barrier is determined by the two equations $g(\theta)=\theta b^{\prime}(T)$ and $g^{\prime}(\theta)=b(T) / T$, where $g(\theta)$ is the cumulant generating function of the increments (and a prime denotes differentiation). The rate function is $R(s)=\theta b(s)-g(\theta) s$, where $\theta$ is determined by the equation $g^{\prime}(\theta)=b(s) / s$.

The exponential rate of decay is in line with what one expects from the results reported in insurance literature. Furthermore, the rate function of the first passage time is convex and $R(s)>0$ for all $s$ if $\mu s<b(s)$, where $\mu$ is the expected value of $X_{i}$. If there is a $T$ such that $\mu T=b(T)$, then $R(s)$ is no longer convex and $R(T)=0$.

The Esscher transform tilts the true distribution $\mathrm{P}$ to a distribution $\mathrm{P}_{\theta}$, so that the drift changes. By an appropriate choice of $\theta$, the drift is changed to the tilted drift $\mu_{\theta}=b(t) / t$ (and $\mu_{\theta}=b(T) / T$ for $\left.t \geq T\right)$. The variance of the increments is denoted by $\sigma^{2}$.

Let $\stackrel{\mathrm{P}_{\theta}}{\rightarrow}$, denote convergence in probability under the tilted measure. Under the tilted distribution, we use large deviations to prove four properties of the stopping time. Three of them have corresponding results in nonlinear renewal theory, namely,

1. $\lim _{c \rightarrow \infty} c^{-1 / 2}\left(N_{c}-c t\right) \stackrel{\mathrm{P}_{\theta}}{\rightarrow} Y \sim N\left(0, a^{2}\right)$, where $a^{2}=\sigma^{2} t^{3}\left(b(t)-b^{\prime}(t) t\right)^{-2}$ and $g^{\prime}(\theta)=b(t) / t$

2. the overshoot $Z$ is asymptotically independent of $Y$ under the tilted distribution $\mathrm{P}_{\theta}$; and

3. the overshoot $Z$ converges in distribution to an overshoot $Z_{l}$ over a linear barrier $c b(t)+$ $b^{\prime}(t)(n-c t)$ under $\mathrm{P}_{\theta}$.

These results, and the techniques used in their proofs, differ from the ones in nonlinear renewal theory in that the barrier is convex and we use large-deviation techniques. The fourth property is that the overshoot over a linear barrier is bounded by an exponential random variable.

One important use of these properties is to show that

$$
\lim _{c \rightarrow \infty} \mathrm{e}^{c R(T)} \mathrm{P}\left(N_{c} \leq c T+c^{1 / 2} y\right)=\frac{\eta}{a} \mathrm{E}_{\theta}\left[\mathrm{e}^{-\theta Z_{l}}\right] \Phi\left(\frac{y}{\eta}\right),
$$

where $\mathrm{E}_{\theta}[\cdot]$ is the expected value under the measure $\mathrm{P}_{\theta}, \Phi$ is the cumulative distribution function of a standard normal random variable, $Z_{l}$ is the overshoot over the linear barrier $c b(T)+b^{\prime}(T)(n-c T)$ and $\eta^{-2}=a^{-2}+b^{\prime \prime}(T) \theta$. 
Example 3. Let $X_{i} \sim N(0,1)$ and $b(s)=1+s^{2}$. Then $g(\theta)=\frac{1}{2} \theta^{2}$, which implies that the system of equations to be solved is

$$
\frac{1}{2} \theta^{2}=2 \theta T, \quad \theta=\frac{1+T^{2}}{T} .
$$

The solution is $\theta=4 / 3^{1 / 2}$ and $T=3^{-1 / 2}$. Hence,

$$
R(T)=\theta b(T)-g(\theta) T=\frac{4\left(1+\frac{1}{3}\right)}{\sqrt{3}}-\frac{8}{3 \sqrt{3}}=\frac{8}{3 \sqrt{3}} .
$$

The variance is

$$
a^{2}=\sigma^{2} t^{3}\left(b(T)-b^{\prime}(T) T\right)^{-2}=\frac{1}{4} \sqrt{3}
$$

and $\eta^{-2}=a^{-2}+\theta b^{\prime \prime}(T)=4 / 3^{1 / 2}+2 \cdot 4 / 3^{1 / 2}=12 / 3^{1 / 2}$. If we simply ignore the constant $\mathrm{E}_{\theta}\left[\mathrm{e}^{-\theta Z_{l}}\right]$ then we can write

$$
\mathrm{P}\left(N_{c} \leq c T+c^{1 / 2} y\right) \approx \exp (-c R(T)) \frac{\eta}{a} \Phi\left(\frac{y}{\eta}\right)=\sqrt{3} \exp \left(-\frac{8 c}{3 \sqrt{3}}\right) \Phi\left(\frac{y \sqrt{12}}{\sqrt[4]{3}}\right) .
$$

Furthermore, the constant $\mathrm{E}_{\theta}\left[\mathrm{e}^{-\theta Z_{l}}\right]$ can also be approximated; see [28, p. 175].

Example 4. As a comparison to renewal theory, let $b(s)=1$. Then, the barrier is both convex and concave. Furthermore, assume that there is a $T$ such that $\mu T=1$. This implies that $R(T)=0$, and that the tilted measure is equal to the original one $(\theta=0)$. We have $\mu=1 / T>0$ and, therefore, by Theorem III 5.1 of [10],

$$
\lim _{c \rightarrow \infty} \mathrm{P}\left(\frac{N_{c}-c / \mu}{\sqrt{\sigma^{2} \mu^{-3} c}} \leq y\right)=\lim _{c \rightarrow \infty} \mathrm{P}\left(\frac{N_{c}-c T}{\sqrt{\sigma^{2} T^{3} c}} \leq y\right)=\Phi(y) .
$$

Not surprisingly, this is equal to the expression given in (1).

When $t<T$, the rate function is

$$
\inf _{s \leq t} R(s)=R(t)=\theta b(t)-g(\theta), \quad \text { where } g^{\prime}(\theta)=b(t) / t .
$$

The function that multiplies the exponential part is not so easy to compute in this case. In Section 5 we will argue that

$$
\lim _{c \rightarrow \infty} c^{1 / 2} \mathrm{e}^{c R(t)} \mathrm{P}\left(N_{c} \leq c t\right)=\frac{\mathrm{E}_{\theta}\left[\mathrm{e}^{-\theta Z_{l}}\right]}{\left(1-\mathrm{e}^{R^{\prime}(t)}\right) \sqrt{2 \pi} a},
$$

where $a^{2}=\sigma^{2} t^{3}\left(b(t)-b^{\prime}(t) t\right)^{-2}$ (as above) and $R^{\prime}(t)=\theta b^{\prime}(t)-g(\theta)$. The proof of this can be found in Hammarlid [12].

A unified formulation of the function that multiplies the exponential part for the two cases $t<T$ and $t \geq T$ may possibly be derived as Höglund [14] did in the case of a sum of independent random variables. Another step towards generalization is to apply the method presented here to concave barriers, but a problem here is that the convexity of $R(s)$ is lost and, in an extreme case, there might be multiple candidates for $T$; see Martin-Löf [22].

The outline of the article is as follows. In Section 2, we introduce large-deviation techniques and give a short, heuristic calculation of the limit distribution. Furthermore, we use largedeviation techniques to derive the equations determining the dominating point of the barrier 
and the rate function $R(s)$ and its properties. In Section 3, we discuss the tilting of a distribution and its implications for the rate function and convergence of the normalized stopping. All the results regarding asymptotic normality, independence, and the distribution of the overshoot are proved in Section 4. The results from previous sections are then used in Section 5 to prove the main theorem.

\section{A large-deviation estimate of the first passage time}

In this section, we will compute the counterpart to the rate function of a sum, namely the rate function of the stopping time. First, however, we will give an introduction to large deviations and a short, heuristic explanation of our general argument.

Assume that the cumulant function $g(\theta)=\log \left(\mathrm{E}\left[\mathrm{e}^{\theta X_{i}}\right]\right)$ exists for $\theta$ in some open set, rule out the special case of $X_{i}$ being a constant, meaning that $g^{\prime \prime}(\theta)>0$, and write $\mu$ for the expected value of $X_{i}$. No assumptions are imposed on $\mu$, but the most interesting case is when $\mu s<b(s)$. A simple application of Markov's inequality gives, for $x>\mu$ and $\theta>0$,

$$
\mathrm{P}\left(S_{n}>n x\right) \leq \exp [-n(x \theta-g(\theta))] .
$$

The best possible approximation is achieved by minimizing over $\theta$. Therefore, we define the rate function $I(x)=\sup _{\theta}(x \theta-g(\theta))$ and find that

$$
\mathrm{P}\left(S_{n}>n x\right) \leq \exp (-n I(x)) \text { if } x>\mu .
$$

This bound is called the Chernoff bound [2]. There is also a lower bound such that [1], for any convex set $B$,

$$
\lim _{n \rightarrow \infty} n^{-1} \log \left(\mathrm{P}\left(\frac{S_{n}}{n} \in B\right)\right)=-\inf _{x \in B} I(x) .
$$

This result is usually stated as two separate limit theorems: one upper bound for closed sets, and one lower bound for open sets. We will work with sets, such as intervals and convex sets, such that the upper bound equals the lower bound.

The rate function has some well-known properties:

$$
\begin{aligned}
I(x) & \geq 0 \quad \text { for all } x, \\
I(\mu) & =0 \\
I(x) & =\theta x-g(\theta), \quad \text { where } g^{\prime}(\theta)=x, \\
I^{\prime}(x) & =\theta \\
\theta^{\prime}(x) & =1 / g^{\prime \prime}(\theta)
\end{aligned}
$$

In large-deviation theory, it is customary to suppress the dependence of $\theta$ on $x$. We will adhere to this custom for notational convenience. However, $\theta$ will be fixed by either $x=b(t) / t$ when $t<T$, or $x=b(T) / T$ when $t \geq T$. Whenever necessary, we will write $\theta(x)$. All of the properties follow from manipulations of the definition of rate function; see, for example, [1] and [4].

The intuitive appeal of large-deviation techniques can be explained by the following heuristic argument. Loosely speaking, we could say that the theory of large deviations tells us that $\mathrm{P}\left(S_{n} / n \approx x\right) \approx \exp (-n I(x))$. Hitting the barrier at $n$ implies a drift $S_{n} / n \approx(c / n) b(n / c)$ and a rate $I((c / n) b(n / c))$. Therefore, we expect the following estimate:

$$
\mathrm{P}\left(N_{c} / c \approx s\right) \approx K(c) \exp (-c s I(b(s) / s))=K(c) \exp (-c R(s)),
$$


where

$$
R(s)=s I(b(s) / s)
$$

and $K(c)$ is a function changing more slowly than the exponential term. When the scale parameter is large, the time $T$, defined by $R(T)=\inf _{s} R(s)$, will dominate, so that

$$
\mathrm{P}\left(N_{c} / c<\infty\right) \approx \sum_{s} K(c) \exp (-c R(s)) \approx K(c) \exp (-c R(T))
$$

Furthermore, for $t \geq T$, by the same type of argument, we have

$$
\mathrm{P}\left(N_{c} / c<t\right) \approx K(c) \exp (-c R(T)),
$$

while, for $t<T$, the dominant time is $t$ and $\mathrm{P}\left(N_{c} / c<t\right) \approx K(c) \exp (-c R(t))$.

Lemma 1. Let $\mu=\mathrm{E}\left[X_{i}\right]$ and assume that $\mu s<b(s)$ for all $s$. Then the function $R(s)=$ $s I(b(s) / s)$ is convex and it attains its minimum at $T$, which is determined by

$$
g(\theta)=b^{\prime}(T) \theta \quad \text { and } \quad g^{\prime}(\theta)=b(T) / T .
$$

The rate function of the stopping time is $R(s)=\theta b(s)-g(\theta) s$, where $g^{\prime}(\theta)=b(s) / s$. Therefore,

$$
\lim _{c \rightarrow \infty} c^{-1} \log \left(\mathrm{P}\left(N_{c} \leq c t\right)\right)= \begin{cases}-R(t) & \text { for } t<T, \\ -R(T) & \text { for } t \geq T .\end{cases}
$$

Remark 1. By (4), is it possible to write $R(T)=\theta\left(b(T)-T b^{\prime}(T)\right)=T\left(\theta g^{\prime}(\theta)-g(\theta)\right)$.

Proof of Lemma 1. The proof is carried out in two steps. First, we show that $c^{-1} \log \left(\mathrm{P}\left(N_{c} \leq\right.\right.$ $c t)$ ) has an upper and a lower bound which, in the limits, are equal. Then, the first- and secondorder derivatives of $R(s)$ are computed, where $R^{\prime \prime}(s) \geq 0$ and the unique solution to $R^{\prime}(s)=0$ is the optimal time $T$.

The probability we want to estimate is

$$
\mathrm{P}\left(N_{c} \leq c t\right)=\mathrm{P}\left(\bigcup_{n=1}^{\lfloor c t\rfloor}\left\{S_{n} \geq c b\left(\frac{n}{c}\right)\right\}\right),
$$

where $\lfloor\cdot\rfloor$ denotes the integer part. This probability has an upper bound, derived from the Chernoff bound (2) and Boole's inequality. Since $(c / n) b(n / c)>\mu$ for all $n$, we have

$$
\mathrm{P}\left(\bigcup_{n=1}^{\lfloor c t\rfloor}\left\{S_{n} \geq c b\left(\frac{n}{c}\right)\right\}\right) \leq \sum_{n=1}^{\lfloor c t\rfloor} \exp \left(-c\left(\frac{n}{c}\right) I\left(\frac{c}{n} b\left(\frac{n}{c}\right)\right) .\right.
$$

One point will dominate the others in the sum. Hence, if $s=n / c$, then

$$
\mathrm{P}\left(\bigcup_{n=1}^{\lfloor c t\rfloor}\left\{S_{n} \geq c b\left(\frac{n}{c}\right)\right\}\right) \leq c t \exp \left(-c \inf _{0 \leq s \leq t} s I\left(\frac{b(s)}{s}\right)\right) .
$$

Let us turn to the lower bound of $\mathrm{P}\left(N_{c} \leq c t\right)$. It is always true that, for any $n \leq c t$,

$$
\mathrm{P}\left(\frac{S_{n}}{n} \geq \frac{c}{n} b\left(\frac{n}{c}\right)\right) \leq \mathrm{P}\left(\bigcup_{n=1}^{\lfloor c t\rfloor}\left\{S_{n} \geq c b\left(\frac{n}{c}\right)\right\}\right) .
$$


Therefore, if we fix $s$ such that $s=n / c$, the lower large-deviation bound ensures that

$$
-s I(b(s) / s) \leq \lim _{c \rightarrow \infty} c^{-1} \log \left(\mathrm{P}\left(S_{n} / n \geq(c / n) b(n / c)\right)\right), \quad s=n / c .
$$

The $s$ that maximizes the rate on the left-hand side of (6) gives the best bound and is equal to the dominant point $T$ in (5). Take the logarithm of both sides of (5) and divide by $c$. Then, in the limit, and in combination with the lower bound (6), we have

$$
\lim _{c \rightarrow \infty} c^{-1} \log \left(\mathrm{P}\left(N_{c} \leq c t\right)\right)=-\inf _{0 \leq s \leq t} s I(b(s) / s)=-\inf _{0 \leq s \leq t} R(s) .
$$

The second step of the proof is to compute the first- and second-order derivatives of $R(s)$ in order to find the unique optimal point. The first-order derivative is $R^{\prime}(s)=I(b(s) / s)+$ $I^{\prime}(b(s) / s)\left[b^{\prime}(s) s-b(s)\right] / s$, which we simplify, using the properties of the rate function (3), to

$$
R^{\prime}(s)=\theta b^{\prime}(s)-g(\theta) \text {. }
$$

Without restrictions on $s$, the minimum of $R(s)$ is attained when $R^{\prime}(s)=0$ and, hence, by (7), we have $b^{\prime}(T) \theta=g(\theta)$, where $g^{\prime}(\theta)=b(T) / T$.

The second-order derivative of $R(s)$ is, from (7), $R^{\prime \prime}(s)=(\mathrm{d} / \mathrm{d} s)\left(\theta b^{\prime}(s)-g(\theta)\right)$. Using the chain rule and the fact that $\theta^{\prime}(x)=1 / g^{\prime \prime}(\theta)$, and making the substitution $g^{\prime}(\theta)=b(s) / s$, gives

$$
R^{\prime \prime}(s)=\frac{\left(b^{\prime}(s) s-b(s)\right)^{2}}{g^{\prime \prime}(\theta) s^{3}}+\theta b^{\prime \prime}(s) .
$$

The barrier and the cumulant function are convex and, hence, $b^{\prime \prime}(s) \geq 0$ for all $s$ and $g^{\prime \prime}(\theta)>0$ for all $\theta$ in the defined open set. We know that $g^{\prime}(\theta)$ is nondecreasing, since $g^{\prime \prime}(\theta)>0$, and that $g^{\prime}(0)=\mu$. This implies that the value of $\theta$ that solves $g^{\prime}(\theta)=b(s) / s$ must be greater than 0 when $b(s) / s>\mu$ and less than 0 when $b(s) / s<\mu$. Therefore, since $b(s) / s>\mu$ for all $s$ and $s>0$, we have $R^{\prime \prime}(s) \geq 0$.

If $t<T$ then the optimal solution is not feasible, and the solution must be on the boundary of $[0, t)$. The function $R(s)$ is decreasing in $s \leq T$, so inf ${ }_{s \leq t} R(s)=R(t)$.

Remark 2. Note that if $s=T$ in (8) then, by Remark 1 ,

$$
R^{\prime \prime}(T)=\frac{R(T)^{2}}{g^{\prime \prime}(\theta) \theta^{2} T^{3}}+\theta b^{\prime \prime}(T) .
$$

Every point $s>T^{*}$, where $T^{*}$ solves $b^{\prime}(s)=b(s) / s$ and may be infinite, is on the 'shadow side'. We call it the shadow side because no point on that side can be reached by a straight line from the origin without passing the barrier along the way. The barrier is convex and therefore

$$
b(s) / s>b^{\prime}(s) \text { for every } s<T^{*} .
$$

When the barrier is crossed by the drift line, the function $R(s)$ is no longer convex.

Lemma 2. Assume that there is a $T$ that solves $\mu T=b(T)$. Then $R(T)=R^{\prime}(T)=0$ and the function $R(s)$ is not, in general, convex. More precisely, $R^{\prime \prime}(s)>0$ for all $s \leq T<T^{*}$, and $R^{\prime \prime}\left(T^{*}\right) \leq 0$. 
Proof. The minimum of $I(\mu)$ is 0 , by the properties of the rate function (3). This, and the assumption that $\mu T=b(T)$, gives $R(T)=T I(b(T) / T)=0$. We know that $\theta=0$ solves $g^{\prime}(\theta)=b(T) / T$, which, by (7), gives $R^{\prime}(T)=0$. When $s \leq T<T^{*}, b(s) / s \geq \mu$, which implies that $\theta \geq 0$ and, therefore, that $R^{\prime \prime}(s)>0$ (see the discussion under (8)). On the other hand, $\theta<0$ when $g^{\prime}(\theta)=b\left(T^{*}\right) / T^{*}<\mu$ and $R^{\prime \prime}\left(T^{*}\right)=\theta b^{\prime \prime}\left(T^{*}\right) \leq 0$. Hence, the function $R(s)$ is not convex.

Remark 3. How is the value of the rate function at the point $u<T^{*}$ related to that at the point $v>T^{*}$, when $u$ and $v$ are the crossing times of a line from the origin to the barrier $b(s)$ ? The relation is linear, because the slope of the line is the same at each point. That is, $b(v) / v=b(u) / u$ and

$$
v I(b(v) / v)=u I(b(u) / u)+(v-u) I(b(u) / u) .
$$

From this, we see that $T \leq T^{*}$.

\section{Tilted distribution}

One important tool is the tilted distribution - sometimes called an exponential change of measure or the Esscher transform; see, for example, [1] and [22]. For every $\theta$ in the defined open set, a tilting of a distribution $F(x)$ is defined by $\mathrm{d} F_{\theta}(x)=\exp (\theta x-g(\theta)) \mathrm{d} F(x)$. The tilted expectation is denoted $\mathrm{E}_{\theta}[\cdot]$ and the tilted cumulant function $g_{\theta}(\gamma)=\log \left(\mathrm{E}_{\theta}\left[\exp \left(\gamma X_{i}\right)\right]\right)$. It can easily be shown that $g_{\theta}(\gamma)=g(\gamma+\theta)-g(\theta)$. The expected value and variance under the tilted measure are therefore defined by the first- and second-order derivatives of the tilted cumulant at $\gamma=0$, as follows:

$$
\mathrm{E}_{\theta}\left[X_{i}\right]=g^{\prime}(\theta) \text { and } \operatorname{var}_{\theta}\left(X_{i}\right)=g^{\prime \prime}(\theta) .
$$

The tilted rate function is $I_{\theta}(x)=\gamma x-g_{\theta}(\gamma)$, where $\gamma$ solves $g_{\theta}^{\prime}(\gamma)=x$, and $I_{\theta}\left(g^{\prime}(\theta)\right)=0$ since $g^{\prime}(\theta)=\mathrm{E}_{\theta}\left[X_{i}\right]$. Also, $I_{\theta}^{\prime}\left(g^{\prime}(\theta)\right)=0$ since $I_{\theta}^{\prime}(x)=\gamma$, according to the properties of the rate function (3), and $\gamma=0$ solves $g_{\theta}^{\prime}(\gamma)=g^{\prime}(\theta)=\mathrm{E}_{\theta}\left[X_{i}\right]=\mu_{\theta}$.

Lemma 3. Let $t \leq T^{*}$ be fixed and let $\theta$ solve $g^{\prime}(\theta)=b(t) / t$. Then the function $R_{\theta}(s)=$ $s I_{\theta}(b(s) / s)$ is not, in general, convex, but $R_{\theta}(t)=0, R_{\theta}^{\prime}(t)=0$, and $R_{\theta}^{\prime \prime}(t)>0$. Furthermore, there is a constant $d>0$ such that $R_{\theta}(t \pm \varepsilon) \leq d \varepsilon^{2}$ for some fixed $\varepsilon>0$. In particular,

$$
\begin{aligned}
& \mathrm{P}_{\theta}\left(N_{c}<c(t-\varepsilon)\right) \leq c t \mathrm{e}^{-c R_{\theta}(t-\varepsilon)} \leq c t \mathrm{e}^{-c d \varepsilon^{2}}, \\
& \mathrm{P}_{\theta}\left(N_{c}>c(t+\varepsilon)\right) \leq \mathrm{e}^{-c R_{\theta}(t+\varepsilon)} \leq \mathrm{e}^{-c d \varepsilon^{2}} .
\end{aligned}
$$

Remark 4. The most important case is when $t=T$.

Remark 5. Only $c t \geq 1$ is of interest since, trivially, $\mathrm{P}\left(N_{c}<1\right)=0$.

Remark 6. In analogy with the case of a linear barrier, it is tempting to believe that, for any fixed $c$, we have $\mathrm{P}_{\theta}\left(N_{c}<\infty\right)=1$. This is not always true, however: it is only true when $b^{\prime}(s) \leq \mu_{\theta} s=s b(t) / t$ for all $s$.

Assume that there is a $T^{* *}$ such that $b^{\prime}(s)>\mu_{\theta} s$ for all $s \geq T^{* *}$. Then, for a fixed $c$, there is a strictly positive probability of not passing the barrier before $T^{* *}$. The probability of not passing the barrier after $T^{* *}$ is also positive since the process, from this point on, may follow the drift without passing the barrier. Hence, the probability in this case is $\mathrm{P}_{\theta}\left(N_{c}<\infty\right)<1$. 
Proof of Lemma 3. The tilted drift is $\mu_{\theta}=g^{\prime}(\theta)=b(t) / t$, which crosses the barrier at time $t$. The minimum of $R_{\theta}(t)$ and $R_{\theta}^{\prime}(t)$ is 0 and $R_{\theta}(t)$ is not generally convex but $R_{\theta}^{\prime \prime}(t)>0$ (all of which follow from Lemma 2). Therefore, the Taylor expansion of $R_{\theta}(t \pm \varepsilon)$ is $\frac{1}{2} R_{\theta}^{\prime \prime}(\xi) \varepsilon^{2}$, where $|\xi-t| \leq \varepsilon$, so we choose $d \leq \frac{1}{2} R_{\theta}^{\prime \prime}(s)$ for all $|s-t| \leq \varepsilon$.

Now consider the sets

$$
\begin{aligned}
& \left\{N_{c}<c(t-\varepsilon)\right\}=\left\{S_{n} \geq c b(n / c) \text { for some } n<c(t-\varepsilon)\right\} \\
& \left\{N_{c}>c(t+\varepsilon)\right\}=\left\{S_{n}<c b(n / c) \text { for all } n \leq c(t+\varepsilon)\right\} .
\end{aligned}
$$

To the probability of the first set we apply Boole's inequality and then the Chernoff bound. The second set is a subset of $\left\{S_{c(t+\varepsilon)}<c b(c(t+\varepsilon)) /(t+\varepsilon)\right\}$, which can be dominated using the Chernoff bound. Hence,

$$
\begin{aligned}
\mathrm{P}_{\theta}\left(S_{n} \geq c b(n / c) \text { for some } n<c(t-\varepsilon)\right) & \leq c t \exp \left(-c \inf _{0 \leq s<t-\varepsilon} s I_{\theta}(b(s) / s)\right), \\
\mathrm{P}_{\theta}\left(S_{n}<c b(n / c) \text { for all } n<c(t+\varepsilon)\right) & \leq \exp \left(-c(t+\varepsilon) I_{\theta}(b(t+\varepsilon) /(t+\varepsilon))\right) .
\end{aligned}
$$

The definition of $R_{\theta}(s)=s I_{\theta}(b(s) / s)$ and the Taylor expansion then give (11).

We write $\stackrel{\text { a.s. }}{\rightarrow}$ for $\mathrm{P}_{\theta}$-almost-sure convergence.

Lemma 4. Fix $\varepsilon>0, \gamma>0$, and $\alpha>0$ such that $\alpha<2 \gamma$. Then, under the tilted distribution, there is a $d>0$ such that

$$
\mathrm{P}_{\theta}\left(\left|\frac{\left(N_{c}-c t\right)^{\alpha}}{c^{\gamma}}\right|>\varepsilon\right) \leq 2 c t \exp \left(-c^{2 \gamma / \alpha-1} d \varepsilon^{2 / \alpha}\right),
$$

where $g^{\prime}(\theta)=b(t) / t$ with $t \leq T$. Also, $c^{-\gamma}\left(N_{c}-c t\right)^{\alpha} \stackrel{\text { a.s. }}{\rightarrow} 0$ as $c \rightarrow \infty$ in the set of integers.

Proof. We have, by the large-deviation estimates of Lemma 3,

$$
\mathrm{P}_{\theta}\left(\left|N_{c} / c-t\right|>\varepsilon\right) \leq 2 c t \mathrm{e}^{-c d \varepsilon^{2}} .
$$

Therefore,

$$
\mathrm{P}_{\theta}\left(\left|\frac{\left(N_{c}-c t\right)^{\alpha}}{c^{\gamma}}\right|>\varepsilon\right)=\mathrm{P}_{\theta}\left(\left|\frac{N_{c}-c t}{c}\right|>c^{\gamma / \alpha-1} \varepsilon^{1 / \alpha}\right) \leq 2 c t \exp \left(-c^{2 \gamma / \alpha-1} d \varepsilon^{2 / \alpha}\right),
$$

where we have used the fact that $c t \geq 1$. If $2 \gamma>\alpha$ then $c t \exp \left(-d \varepsilon^{2 / \alpha} c^{2 \gamma / \alpha-1}\right) \rightarrow 0$ as $c$ goes to infinity. Choose $\varepsilon=c^{-\eta}$, where $\eta>0$ is such that $\gamma-\eta>\frac{1}{2} \alpha$. Then

$$
\sum_{c=1}^{\infty} \mathrm{P}_{\theta}\left(\left|\frac{\left(N_{c}-c t\right)^{\alpha}}{c^{\gamma}}\right|>\frac{1}{c^{\eta}}\right) \leq 2 \sum_{c=1}^{\infty} c t \exp \left(-c^{2(\gamma-\eta) / \alpha-1} d\right)<\infty .
$$

The Borel-Cantelli lemma states that the convergence is almost sure.

Corollary 1. Fix $\varepsilon$. Then there is a constant $d>0$ such that, under the tilted distribution, $\mathrm{P}_{\theta}\left(\left|N_{c}-c T\right|>c \varepsilon\right) \leq 2 c t \mathrm{e}^{-c d \varepsilon^{2}}$, where $g^{\prime}(\theta)=b(T) / T$ and $N_{c} / c \stackrel{\text { a.s. }}{\rightarrow} T$ as $c \rightarrow \infty$ in the set of integers.

Proof. This is a direct consequence of Lemma 4, with $t=T, \gamma=1$, and $\alpha=1$. 
Later, we will need to use the fact that the overshoot $Z$ converges to 0 when scaled by $c^{-\alpha}$. However, if we use the definition of the overshoot, Taylor expand the barrier around the dominating point, and use the fact that $S_{N_{c}-1} \leq c b\left(\left(N_{c}-1\right) / c\right)$, then

$$
Z=S_{N_{c}}-c b\left(N_{c} / c\right) \leq X_{N_{c}}-b^{\prime}(t)+\left(b^{\prime \prime}(\xi) / c\right)\left|N_{c}-1-c t\right|,
$$

where $\xi$ is between $t$ and $N_{c} / c$. The last step (in $S_{n}$ ) might be negative, but the overshoot is, of course, always positive.

Lemma 5. Let $\alpha>0$. Then, under the tilted distribution,

$$
\frac{\left|X_{N_{c}}\right|}{c^{\alpha}} \stackrel{\text { a.s. }}{\rightarrow} 0 \text { as } c \rightarrow \infty .
$$

For a proof, see that of Theorem I.2.3(i) of Gut [10].

Lemma 6. Let $\alpha>0$. Then, under the tilted distribution,

$$
\frac{Z}{c^{\alpha}} \stackrel{\text { a.s. }}{\rightarrow} 0 \quad \text { as } c \rightarrow \infty \text {. }
$$

Proof. This is a direct consequence of (12), Lemma 5, and the fact that $\lim _{c \rightarrow \infty} b^{\prime \prime}(\xi)$ is an almost-everywhere-bounded random variable, so that $c^{-(1+\alpha)} b^{\prime \prime}(\xi)\left|N_{c}-1-c t\right| \stackrel{\text { a.s. }}{\rightarrow} 0$ (by Lemma 4).

\section{Normality, overshoot, and independence}

\subsection{Asymptotic normality}

Gut [9], [10] uses Anscombe's theorem as a key factor in proving asymptotic normality for stopping times of random walks or perturbed random walks hitting a regularly varying, linear or concave boundary. We will use the same technique.

Theorem 1. (Anscombe's theorem.) Let $\left\{X_{i}, i \geq 1\right\}$ be a sequence of independent, identically distributed random variables with mean 0 and variance $\sigma^{2}$ and let $\left\{S_{n}, n \geq 1\right\}$ denote their partial sums. Furthermore, assume that $M_{c}$ is a sequence of integer-valued, positive random variables such that $M_{c} / c \stackrel{\mathrm{P}_{\theta}}{\rightarrow} T$ as $c \rightarrow \infty$. Then

$$
\begin{aligned}
& \lim _{c \rightarrow \infty} \mathrm{P}\left(S_{M_{c}} \leq y \sigma \sqrt{M_{c}}\right)=\Phi(y), \\
& \lim _{c \rightarrow \infty} \mathrm{P}\left(S_{M_{c}} \leq y \sigma \sqrt{c T}\right)=\Phi(y) .
\end{aligned}
$$

Proof. For a proof, see Rényi [23] or Gut [10].

Theorem 2. Assume that $\operatorname{var}_{\theta}\left(X_{i}\right)=\sigma^{2}=g^{\prime \prime}(\theta)<\infty$ and that $g^{\prime}(\theta)=b(t) / t$. Then, for $t \leq T^{*}$,

$$
\lim _{c \rightarrow \infty} \mathrm{P}_{\theta}\left(\frac{b(t)-b^{\prime}(t) t}{t}\left(N_{c}-c t\right) \leq y \sigma \sqrt{c t}\right)=\Phi(y) .
$$

In the special case in which $g^{\prime}(\theta)=b(T) / T$, we have $b(T)-b^{\prime}(T) T=\theta^{-1} R(T)$ and

$$
\lim _{c \rightarrow \infty} \mathrm{P}_{\theta}\left(\frac{R(T)}{\theta T}\left(N_{c}-c T\right) \leq y \sigma \sqrt{c T}\right)=\Phi(y) .
$$


Proof. Start with Anscombe's theorem,

$$
\lim _{c \rightarrow \infty} \mathrm{P}_{\theta}\left(S_{N_{c}}-g^{\prime}(\theta) N_{c} \leq y \sigma \sqrt{c t}\right)=\Phi(y) .
$$

The stopped process is, by Definition 1, greater than or equal to the barrier; hence,

$$
\frac{c b\left(N_{c} / c\right)-g^{\prime}(\theta) N_{c}}{\sigma \sqrt{c t}} \leq \frac{S_{N_{c}}-g^{\prime}(\theta) N_{c}}{\sigma \sqrt{c t}} \leq \frac{c b\left(N_{c} / c\right)-g^{\prime}(\theta) N_{c}}{\sigma \sqrt{c t}}+\frac{Z}{\sigma \sqrt{c t}} .
$$

The last term on the right-hand side converges to 0 in probability, by Lemma 6 . Substituting the Taylor expansion

$$
c b\left(N_{c} / c\right)=c b(t)+b^{\prime}(t)\left(N_{c}-c t\right)+\frac{1}{2} c^{-1} b^{\prime \prime}(\xi)\left(N_{c}-c t\right)^{2},
$$

where $\xi$ is between $t$ and $N_{c} / c$, into (13), and using the fact that $g^{\prime}(\theta)=b(t) / t$, gives

$$
\begin{aligned}
\frac{c b\left(N_{c} / c\right)-g^{\prime}(\theta) N_{c}}{\sigma \sqrt{c t}} & =\frac{c b(t)+b^{\prime}(t)\left(N_{c}-c t\right)+\frac{1}{2} c^{-1} b^{\prime \prime}(\xi)\left(N_{c}-c t\right)^{2}-N_{c} b(t) / t}{\sigma \sqrt{c t}} \\
& =-\frac{\left(b(t)-b^{\prime}(t) t\right)\left(N_{c}-c t\right)}{\sigma t \sqrt{c t}}+\frac{b^{\prime \prime}(\xi)\left(N_{c}-c t\right)^{2}}{2 c^{3 / 2} t^{1 / 2} \sigma} .
\end{aligned}
$$

When $s \leq T^{*}, b^{\prime \prime}(s)$ is bounded and $c^{-3 / 2}\left(N_{c}-c t\right)^{2} \stackrel{\mathrm{P}_{\theta}}{\rightarrow} 0$ as $c$ goes to infinity, by Lemma 4 . Hence, the term quadratic in $N_{c}-c t$ converges to 0 . The theorem then follows by the symmetry of the normal distribution. In the special case in which $g^{\prime}(\theta)=b(T) / T$, we have, by Remark 1 ,

$$
b(T)-b^{\prime}(T) T=R(T) / \theta .
$$

\subsection{Overshoot}

In this section, we show that the overshoot is distributed as is an overshoot over a linear barrier. In our case, the linear barrier of interest is

$$
c l(n / c)=c b(t)+b^{\prime}(t)(n-c t) .
$$

Let

$$
\begin{aligned}
& N_{+}=\inf \left\{n: n \geq 1, S_{n}-n b^{\prime}(t)>0\right\}, \\
& M_{c}=\inf \left\{m: S_{m} \geq c l(m / c)\right\},
\end{aligned}
$$

with possibly infinite stopping times if the process never passes or hits the barrier. However, $\mathrm{P}_{\theta}\left(M_{c}<\infty\right)=1$ and $\mathrm{P}_{\theta}\left(N_{+}<\infty\right)=1$; see [10]. Define the linear overshoot as

$$
Z_{l}=S_{M_{c}}-\operatorname{cl}\left(M_{c} / c\right) .
$$

Now, $Z_{l}$ has a well-known asymptotic distribution $Q_{\theta}(z)$, where $g^{\prime}(\theta)=b(t) / t$, when $c \rightarrow \infty$. This distribution can be expressed in terms of the distribution of $S_{N_{+}}-b^{\prime}(t) N_{+}$; see [28, Corollary 8.33, p. 171].

It is reasonable to believe that $g_{\theta}^{Z_{l}}(\gamma)=\log \left(\mathrm{E}\left[\exp \left(\gamma Z_{l}\right)\right]\right)$ is bounded when $|\gamma|<\bar{\gamma}$, since $g_{\theta}(\gamma)$ exists. It is fairly easy to show that, for an overshoot $Z$ over any convex barrier, we have $\exp \left(g_{\theta}^{Z}(\gamma)\right) \leq c t \exp \left(g_{\theta}(\gamma)\right)$. To prove this, we could use the reasoning leading to (14), below, but instead we strengthen the result as follows. 
Lemma 7. There is a $\bar{\gamma}>0$ such that $g_{\theta}^{Z_{l}}(\gamma)<$ constant, uniformly in $c$, for $\gamma<\bar{\gamma}$.

Proof. First, note that if we define $\tilde{X}_{i}=X_{i}-b^{\prime}(t)$ and $\tilde{S}_{n}=\sum_{i=1}^{n} \tilde{X}_{i}$, the overshoots are equal, i.e.

$$
\tilde{Z}_{l}=\tilde{S}_{M_{c}}-c\left(b(t)-b^{\prime}(t) t\right)=S_{M_{c}}-c l\left(M_{c} / c\right)=Z_{l} .
$$

The expected value $\mathrm{E}_{\theta}\left[\tilde{X}_{i}\right]=b(t) / t-b^{\prime}(t)$ is greater than 0 since $t<T^{*}$, which implies that $\mathrm{E}_{\theta}\left[N_{+}\right]<\infty$. Let us write $\tilde{X}_{i}^{+}=\max \left(\tilde{X}_{i}, 0\right)$ and conclude that

$$
\mathrm{E}_{\theta}\left[\exp \left(\gamma \tilde{X}_{N_{+}}\right)\right] \leq \mathrm{E}_{\theta}\left[\sum_{i=1}^{N_{+}} \exp \left(\gamma \tilde{X}_{i}^{+}\right)\right]=\mathrm{E}_{\theta}\left[N_{+}\right] \mathrm{E}_{\theta}\left[\exp \left(\gamma \tilde{X}_{1}^{+}\right)\right]<\infty .
$$

For a more extensive discussion of this step, see Section I.8 of [10]. Trivially, we have $\tilde{S}_{N_{+}} \leq \tilde{X}_{N_{+}}$and, thus, $g_{\theta}^{+}(\gamma)=\log \left(\mathrm{E}_{\theta}\left[\exp \left(\gamma \tilde{S}_{N_{+}}\right)\right]\right)<\infty$.

Assume that $b(t)-b^{\prime}(t) t=1$, without any loss of generality. Define the sequence of ladder variables as $W_{k}=\sum_{j=1}^{k} \Delta \tilde{S}(j)$, where $\Delta \tilde{S}(j)$ are the independent and identically distributed differences between the old and the new maxima. The number of epochs needed to pass the constant barrier is denoted by

$$
K=\inf _{0 \leq k}\left\{k: W_{k} \geq c\right\} .
$$

We are now prepared to derive the moment-generating function of the linear overshoot. First, split the sample space into the disjoint sets $\{K=k\}$. Then

$$
\begin{aligned}
\mathrm{E}_{\theta}\left[\exp \left(\gamma \tilde{Z}_{l}\right)\right] & =\sum_{k=1}^{\infty} \mathrm{E}_{\theta}\left[\exp \left(\gamma \tilde{Z}_{l}\right), K=k\right] \\
& =\sum_{k=1}^{\infty} \mathrm{E}_{\theta}\left[\mathrm{E}_{\theta}\left[\exp \left(\gamma \tilde{Z}_{l}\right), W_{k-1}<c \leq W_{k} \mid W_{k-1}\right]\right] .
\end{aligned}
$$

The overshoot is $\tilde{Z}_{l}=\Delta \tilde{S}(K)-c+W_{K-1}$ and the distributions of $W_{k}$ and $\Delta \tilde{S}(j)$, which is distributed as is $S_{N_{+}}$, are written $F_{\theta}^{k *}(\mathrm{~d} w)$ and $F_{\theta}(\mathrm{d} s)$, respectively. Therefore,

$$
\mathrm{E}_{\theta}\left[\exp \left(\gamma \tilde{Z}_{l}\right)\right]=\sum_{k=1}^{\infty} \int_{w<c} \int_{s \geq c-w} \exp (\gamma(s-c+w)) F_{\theta}(\mathrm{d} s) F_{\theta}^{(k-1) *}(\mathrm{~d} w) .
$$

If we extend the range of integration of $s$ to the positive half-line, the integral is equal to $\exp \left(g_{\theta}^{+}(\gamma)\right)$. Therefore, we get an upper bound

$$
\begin{aligned}
\mathrm{E}_{\theta}\left[\exp \left(\gamma \tilde{Z}_{l}\right)\right] & \leq \exp \left(g_{\theta}^{+}(\gamma)\right) \sum_{k=1}^{\infty} \int_{w<c} \exp (-\gamma(c-w)) F_{\theta}^{(k-1) *}(\mathrm{~d} w) \\
& =\exp \left(g_{\theta}^{+}(\gamma)\right) \int_{w<c} \exp (-\gamma(c-w)) U(\mathrm{~d} w),
\end{aligned}
$$

where $U(\mathrm{~d} w)$ is the renewal measure; see [10] or [28]. This integral is bounded, which follows by the key renewal theorem [10].

Let us now turn to the overshoot $Z=S_{N_{c}}-c b\left(N_{c} / c\right)$. Our aim is to show that the overshoot converges in distribution to the overshoot over the linear barrier $c l(n / c)$. This could be proved 
by a transformation to a perturbed random walk, using the results of [28], and then transforming back. In doing so, however, the intuition and simplicity of the idea would be lost.

We will make use of the auxiliary stopping time $M_{c-c^{\alpha}}$ and $\Delta N=N_{c}-M_{c-c^{\alpha}}$. The choice of the constant $\alpha>0$ will later be such that the distance left to the true barrier can be neglected in the limit on the scale $c$, but great enough for the overshoot to gain the asymptotic properties.

The overshoot over the auxiliary barrier $\left(c-c^{\alpha}\right) l\left(n /\left(c-c^{\alpha}\right)\right)$ is denoted by $Z_{\alpha}$, and

$$
\begin{aligned}
\mathrm{P}_{\theta}\left(N_{c}=M_{c-c^{\alpha}}\right) & \leq \mathrm{P}_{\theta}\left(Z_{\alpha}>c^{\alpha}\left(b(t)-b^{\prime}(t) t\right)\right) \\
& \leq \exp \left(-c^{\alpha} \gamma\left(b(t)-b^{\prime}(t) t\right)+g_{\theta}^{Z_{\alpha}}(\gamma)\right),
\end{aligned}
$$

where $\gamma>0$ is such that $g_{\theta}^{Z_{\alpha}}(\gamma)=\log \left(\mathrm{E}_{\theta}\left[\exp \left(\gamma Z_{\alpha}\right)\right]\right)<\infty$, by Lemma 7 . Furthermore, $b(t)-b^{\prime}(t) t>0$ for all $t \leq T^{*}$, which follows from (10).

Lemma 8. Assume that $0<\alpha<1$. Then, under the tilted distribution for arbitrary $0<\delta<$ $b(t)-b^{\prime}(t) t$, there are a time $\hat{t} \leq T^{*}$ and a constant $d>0$ such that

$$
\mathrm{P}_{\theta}\left(\left|\Delta N-c^{\alpha} \hat{t}\right|>c^{\alpha} \delta\right) \leq 3 c^{\alpha} \hat{t} \exp \left(-c^{\alpha} d \delta^{2}\right),
$$

where $g^{\prime}(\theta)=b(t) / t$ and $c^{-\alpha} \Delta N \stackrel{\text { a.s. }}{\rightarrow} t$ as $c \rightarrow \infty$ in the set of integers.

Proof. The idea of the proof is that, when the auxiliary barrier is passed, the remaining distance is of order $c^{\alpha}$ and, consequently, so is the remaining time.

Consider the set $\left\{\omega:\left|\Delta N-c^{\alpha} \hat{t}\right|>c^{\alpha} \delta\right\}=A^{\prime} \cup A^{\prime \prime}$, where

$$
\begin{aligned}
& A^{\prime}=\left\{S_{n} \geq c b(n / c) \text { for some } n \leq M_{c-c^{\alpha}}+c^{\alpha}(\hat{t}-\delta)\right\}, \\
& A^{\prime \prime}=\left\{S_{n}<c b(n / c) \text { for all } n \leq M_{c-c^{\alpha}}+c^{\alpha}(\hat{t}+\delta)\right\},
\end{aligned}
$$

and let us write $\Delta n=n-M_{c-c^{\alpha}}$, when $n \geq M_{c-c^{\alpha}}$, and $\Delta S_{n}=S_{n}-S_{M_{c-c^{\alpha}}}$. We assume that $Z_{\alpha} \leq c^{\alpha} \delta$, to ensure that $M_{c-c^{\alpha}}<N_{c}$ and that the remaining distance is of order $c^{\alpha}$. The partial sum may, in this notation, be written as

$$
\begin{aligned}
S_{n} & =\Delta S_{n}+S_{M_{c-c^{\alpha}}} \\
& =\Delta S_{n}+\left(c-c^{\alpha}\right) l\left(M_{c-c^{\alpha}} /\left(c-c^{\alpha}\right)\right)+Z_{\alpha},
\end{aligned}
$$

and the remaining distance is $c b(n / c)-S_{M_{c-c^{\alpha}}}$. By Taylor expanding the original barrier around $t$, we find that the remaining distance is

$$
\begin{aligned}
c b(n / c)-S_{M_{c-c^{\alpha}}} & =c^{\alpha} l\left(\Delta n / c^{\alpha}\right)+\frac{1}{2}\left(b^{\prime \prime}(\xi) / c\right)(n-c t)^{2}-Z_{\alpha} \\
& =c^{\alpha} l\left(\Delta n / c^{\alpha}\right)+O\left((n-c t)^{2} / c+Z_{\alpha}\right),
\end{aligned}
$$

where $\xi$ is between $t$ and $n / c$. We then have

$$
\begin{aligned}
& A^{\prime}=\left\{\Delta S_{n} \geq c^{\alpha} l\left(\Delta n / c^{\alpha}\right)+O\left((n-c t)^{2} / c+Z_{\alpha}\right) \text { for some } \Delta n \leq c^{\alpha}(\hat{t}-\delta)\right\}, \\
& A^{\prime \prime}=\left\{\Delta S_{n}<c^{\alpha} l\left(\Delta n / c^{\alpha}\right)+O\left((n-c t)^{2} / c+Z_{\alpha}\right) \text { for all } \Delta n \leq c^{\alpha}(\hat{t}+\delta)\right\} .
\end{aligned}
$$

The most probable time to hit or cross $c^{\alpha} l\left(\Delta n / c^{\alpha}\right)+O\left((n-c t)^{2} / c+Z_{\alpha}\right)$ is when the barrier is crossed by the drift line $g^{\prime}(\theta) \Delta n$. The most probable time $\hat{t}=\Delta n / c^{\alpha}$ is the solution to

$$
g^{\prime}(\theta) \Delta n / c^{\alpha}=b(t)+b^{\prime}(t)\left(\Delta n / c^{\alpha}-t\right)+O\left((n-c t)^{2} / c^{1+\alpha}+Z_{\alpha} / c^{\alpha}\right) .
$$


The equation

$$
g^{\prime}(\theta) \hat{t}=b(t)+b^{\prime}(t)(\hat{t}-t)+O\left((n-c t)^{2} / c^{1+\alpha}+Z_{\alpha} / c^{\alpha}\right)
$$

always has a solution when $c$ is large enough, because $t \leq T^{*}$ and, therefore, $g^{\prime}(\theta)=b(t) / t>$ $b^{\prime}(t)$ and $b(0)>0$; see the implication (10) of not being on the shadow side. The overshoot term in the remainder converges almost surely to 0 , by Lemma 6. Also, the quadratic term converges in probability to 0 since $M_{c-c^{\alpha}} \leq n \leq N_{c}$ and

$$
c^{-(1+\alpha)}(n-c t)^{2} \leq c^{-(1+\alpha)}\left(\left(M_{c-c^{\alpha}}-c t\right)^{2}+\left(N_{c}-c t\right)^{2}\right),
$$

which, by Lemma 4, converges in probability to 0 . Therefore, when $c \rightarrow \infty$, the solution is $t=\hat{t}$.

Lemma 3, (15), and the law of total probability together imply that

$$
\begin{aligned}
\mathrm{P}_{\theta}\left(\left|\Delta N-c^{\alpha} \hat{t}\right|>c^{\alpha} \delta\right) & \leq \mathrm{P}_{\theta}\left(A^{\prime} \cup A^{\prime \prime}, Z_{\alpha} \leq c^{\alpha} \delta\right)+\mathrm{P}_{\theta}\left(Z_{\alpha}>c^{\alpha} \delta\right) \\
& \leq 3 c^{\alpha} \hat{t} \exp \left(-c^{\alpha} d \delta^{2}\right) .
\end{aligned}
$$

Now, if we put $\delta=c^{-\alpha / 4}$ and sum over $c$, then

$$
\sum_{c=1}^{\infty} \mathrm{P}_{\theta}\left(\left|\Delta N-c^{\alpha} t\right|>\frac{c^{\alpha}}{c^{\alpha / 4}}\right) \leq 3 \sum_{c=1}^{\infty} c^{\alpha} \hat{t} \exp \left(-c^{\alpha / 2} d\right)<\infty .
$$

The Borel-Cantelli lemma ensures the almost-sure convergence.

Lemma 9. Let $0<\alpha<\frac{1}{4}$ and $g^{\prime}(\theta)=b(t) / t$, where $t \leq T^{*}$. Then, under the tilted distribution,

$$
\lim _{c \rightarrow \infty} c^{-1}\left(\left(N_{c}-c t\right)^{2}-\left(M_{c-c^{\alpha}}-\left(c-c^{\alpha}\right) t\right)^{2}\right)=0 \quad \text { almost surely }
$$

in the set of integers.

Proof. The trick of the proof is to split the sample space into two sets; the likely event $A$ and its complement $A^{\mathrm{c}}$. The probability of the unlikely event converges to 0 and, if the likely event occurs, the distance between the two quadratic terms converges to 0 .

Fix an arbitrary $\varepsilon>0$ and $0<\delta<1$ to define the set

$$
A=\left\{\omega:\left|M_{c-c^{\alpha}}-\left(c-c^{\alpha}\right) t\right|<\left(c-c^{\alpha}\right) \varepsilon\right\} \cap\left\{\left|\Delta N-c^{\alpha} \hat{t}\right|<c^{\alpha} \delta\right\} \cap\left\{\left|N_{c}-c t\right|<c \varepsilon\right\} .
$$

On the set $A$ we have, by the conjugate rule, that

$$
\begin{aligned}
\left|\left(N_{c}-c t\right)^{2}-\left(M_{c-c^{\alpha}}-\left(c-c^{\alpha}\right) t\right)^{2}\right| & =\left|N_{c}-M_{c-c^{\alpha}}-c^{\alpha} t\right|\left|N_{c}-c t+M_{c-c^{\alpha}}-\left(c-c^{\alpha}\right) t\right| \\
& \leq c^{\alpha} \delta \varepsilon\left(c+c-c^{\alpha}\right) \\
& \leq 2 c^{\alpha+1} \varepsilon .
\end{aligned}
$$

We choose $\varepsilon=\frac{1}{2} c^{-2 \alpha}$. Then

$$
c^{-1}\left|\left(N_{c}-c t\right)^{2}-\left(M_{c-c^{\alpha}}-\left(c-c^{\alpha}\right) t\right)^{2}\right| \leq c^{-\alpha} \leq \eta \quad \text { when } c>\eta^{-1 / \alpha} \text {, }
$$


which implies that $\mathrm{P}_{\theta}\left(\left|\left(N_{c}-c t\right)^{2}-\left(M_{c-c^{\alpha}}-\left(c-c^{\alpha}\right) t\right)^{2}\right|>c \eta, A\right)=0$. This and the law of total probability give, for $c \geq \eta^{-1 / \alpha}$,

$$
\mathrm{P}_{\theta}\left(\left|\left(N_{c}-c t\right)^{2}-\left(M_{c-c^{\alpha}}-\left(c-c^{\alpha}\right) t\right)^{2}\right|>c \eta\right) \leq \mathrm{P}_{\theta}\left(A^{\mathrm{c}}\right) .
$$

The probability of the complement can be bounded according to Lemma 4 and Lemma 8, as follows:

$$
\begin{aligned}
\mathrm{P}_{\theta}\left(A^{\mathrm{c}}\right) & \leq 3 c^{\alpha} \hat{t} \exp \left(-c^{\alpha} d \delta^{2}\right)+2\left(c-c^{\alpha}\right) t \exp \left(-\left(c-c^{\alpha}\right) c^{-4 \alpha} d\right)+2 c t \exp \left(-c^{1-4 \alpha} d\right) \\
& \leq 4 c t\left(\exp \left(-c^{\alpha} d \delta^{2}\right)+\exp \left(-\left(c-c^{\alpha}\right) c^{-4 \alpha} d\right)\right) .
\end{aligned}
$$

In the last inequality, we have used the fact that $c-c^{\alpha} \leq c$ and assumed that $\hat{t} \leq t$. (However, taking $\hat{t} \geq t$ would cause no practical change.)

Let $\eta=c^{-\alpha / 4}$. Since $\alpha<\frac{1}{4}$, we have $c^{1 / 4 \alpha} \leq c$, which implies the following convergent sum:

$$
\begin{gathered}
\sum_{c=1}^{\infty} \mathrm{P}_{\theta}\left(c^{-1}\left|\left(N_{c}-c t\right)^{2}-\left(M_{c-c^{\alpha}}-\left(c-c^{\alpha}\right) t\right)^{2}\right|>1 / c^{1 / 4}\right) \\
\leq 4 \sum_{c=1}^{\infty} c t\left(\exp \left(-c^{\alpha} d \delta^{2}\right)+\exp \left(-\left(c-c^{\alpha}\right) c^{-4 \alpha} d\right)\right) .
\end{gathered}
$$

The convergence is therefore almost sure, by the Borel-Cantelli lemma.

The idea now is to look at the stopping time of the process to a barrier that is just below the true barrier. The second-order term of this barrier does not change after $M_{c-c^{\alpha}}$, and the corresponding 'auxiliary' stopping time is defined as

$$
N_{l}=\inf \left\{n: S_{n}>\operatorname{cl}(n / c)+b^{\prime \prime}(t)\left(M_{c-c^{\alpha}}-\left(c-c^{\alpha}\right) t\right)^{2} / 2 c-b^{\prime \prime}(t) / 2 c^{\alpha}\right\}
$$

and the corresponding 'auxiliary' overshoot as

$$
\tilde{Z}_{l}=S_{N_{l}}-c l\left(N_{l} / c\right)-b^{\prime \prime}(t)\left(M_{c-c^{\alpha}}-\left(c-c^{\alpha}\right) t\right)^{2} / 2 c+b^{\prime \prime}(t) / 2 c^{\alpha},
$$

where $t \leq T^{*}$. The extra (final) term $b^{\prime \prime}(t) / 2 c^{\alpha}$ is added to ensure that this auxiliary barrier is just below the true barrier on a certain set and, therefore, that $N_{l} \leq N_{c}$.

Lemma 10. When $X_{i}$ is nonarithmetic, under the tilted distribution,

$$
N_{c}-N_{l} \stackrel{\mathrm{P}_{\theta}}{\rightarrow} 0 \quad \text { as } c \rightarrow \infty
$$

where $g^{\prime}(\theta)=b(t) / t$.

When $X_{i}$ is arithmetic with span $h$, the convergence still holds if $c$ does not go to infinity as a multiple of $h$.

Proof. On the set $A$ defined in (16) with $\varepsilon=\frac{1}{2} c^{-2 \alpha}$ and $\alpha<\frac{1}{4}$, we have, for $M_{c-c^{\alpha}} \leq n \leq$ $N_{c}$, that

$$
\frac{b^{\prime \prime}(t)}{2 c}\left((n-c t)^{2}-\left(M_{c-c^{\alpha}}-\left(c-c^{\alpha}\right) t\right)^{2}\right)+\frac{b^{\prime \prime}(t)}{2 c^{\alpha}} \geq \frac{b^{\prime \prime}(t)}{2 c^{\alpha}}-\frac{b^{\prime \prime}(t)}{2 c^{\alpha}}=0,
$$

by the same reasoning that led to (17). Hence, $N_{l} \leq N_{c}$. 
Let us now study the case in which the stopping times are not equal on $A$; that is, where

$$
\begin{aligned}
\left\{\omega: N_{c}>N_{l}\right\} & =\left\{\omega: c l\left(\frac{N_{l}}{c}\right)+\frac{b^{\prime \prime}(t)}{2 c}\left(M_{c-c^{\alpha}}-\left(c-c^{\alpha}\right) t\right)^{2}-\frac{b^{\prime \prime}(t)}{2 c^{\alpha}} \leq S_{N_{l}}<c b\left(\frac{N_{l}}{c}\right)\right\} \\
& =\left\{\omega: 0 \leq \tilde{Z}_{l}<\frac{b^{\prime \prime}(t)}{2 c}\left(\left(N_{l}-c t\right)^{2}-\left(M_{c-c^{\alpha}}-\left(c-c^{\alpha}\right) t\right)^{2}\right)+\frac{b^{\prime \prime}(t)}{2 c^{\alpha}}\right\} \\
& \subseteq\left\{\omega: 0 \leq \tilde{Z}_{l}<\frac{b^{\prime \prime}(t)}{c^{\alpha}}\right\} .
\end{aligned}
$$

By the law of total probability, (15), and the fact that $\lim _{c \rightarrow \infty} \mathrm{P}_{\theta}\left(A^{\mathrm{c}}\right)=0$, we have

$$
\begin{aligned}
\lim _{c \rightarrow \infty} \mathrm{P}_{\theta}\left(N_{c} \neq N_{l}\right) & \leq \lim _{c \rightarrow \infty} \mathrm{P}_{\theta}\left(N_{c}>N_{l}, A\right)+\mathrm{P}_{\theta}\left(A^{\mathrm{c}}\right) \\
& \leq \lim _{c \rightarrow \infty} \mathrm{P}_{\theta}\left(0 \leq Z_{l}<c^{-\alpha} b^{\prime \prime}(t)\right) \\
& =\lim _{c \rightarrow \infty} F_{Z_{l}}^{c}\left(c^{-\alpha} b^{\prime \prime}(t)\right),
\end{aligned}
$$

where $F_{Z_{l}}^{c}(\cdot)$ is the cumulative distribution function of the overshoot, under the tilted measure, for a fixed $c$. The limit distribution $F_{Z_{l}}(\cdot)$ is continuous when $X_{i}$ is nonarithmetic; see [28, Corollary 8.33, p. 171]. Therefore, for fixed $v>0$, there is a $c$ such that $c^{-\alpha} b^{\prime \prime}(t)<v$ and $F_{Z_{l}}^{c}\left(c^{-\alpha} b^{\prime \prime}(t)\right) \leq F_{Z_{l}}^{c}(v)$. We must have $\lim _{c \rightarrow \infty} F_{Z_{l}}^{c}\left(c^{-\alpha} b^{\prime \prime}(t)\right) \leq F_{Z_{l}}(v)$, but $v$ is arbitrary.

When $X_{i}$ is arithmetic with span $h$, the limit distribution of the overshoot is also arithmetic. For $c$ large enough, $c^{-\alpha} b^{\prime \prime}(t)$ is less than the span, and

$$
\lim _{c \rightarrow \infty} \mathrm{P}_{\theta}\left(0 \leq Z_{l}<c^{-\alpha} b^{\prime \prime}(t)\right)=0 .
$$

The one exception is when $c \rightarrow \infty$ as a multiple of $h$. Then the limit is not 0 , but rather $\lim _{c \rightarrow \infty} \mathrm{P}_{\theta}(Z=0)=d / \mathrm{E}\left[S_{N_{+}}\right]$; see [28, Corollary 8.33, p. 171].

From now on we assume that when the $X_{i}$ are arithmetic, $c$ does not go to infinity as a multiple of the span $h$.

Theorem 3. The distribution of the overshoot under the tilted distribution, where $g^{\prime}(\theta)=$ $b(t) / t$ and $t \leq T^{*}$, satisfies

$$
\lim _{c \rightarrow \infty} \mathrm{P}_{\theta}(Z \leq z)=\lim _{c \rightarrow \infty} \mathrm{P}_{\theta}\left(S_{M_{c}}-c l\left(M_{c} / c\right) \leq z\right)=Q_{\theta}(z),
$$

where, recall, $Q_{\theta}(z)$ is the asymptotic distribution of the overshoot $Z_{l}$ over the linear boundary $\mathrm{cl}(n / c)$ and $M_{c}$ is the first passage time to this barrier.

Proof. The idea of the proof is to condition on $M_{c-c^{\alpha}}$ and $S_{M_{c-c^{\alpha}}}$, when and where the process crosses the auxiliary barrier $\left(c-c^{\alpha}\right) l\left(n /\left(c-c^{\alpha}\right)\right)$. This auxiliary barrier is distant enough to preserve the asymptotic properties of the overshoot. Furthermore, when we exchange $N_{c}$ with $M_{c-c^{\alpha}}$ in the second-order term of the expansion of the barrier, the difference vanishes. The real barrier is then exchanged for a linear barrier translated by the curvature of the auxiliary barrier at the stopping time.

In the Taylor expansion of the barrier, the remainder - the third-order term - is ignored because, according to Lemma 4, it converges to 0 in probability under the tilted measure. Thus, $Z=S_{N_{c}}-c b\left(N_{c} / c\right) \stackrel{\mathrm{P}_{\theta}}{\rightarrow} S_{N_{c}}-c l\left(N_{c} / c\right)+\frac{1}{2} c^{-1} b^{\prime \prime}(t)\left(N_{c}-c t\right)^{2}$ when the scale parameter $c$ goes to infinity. The partial sum hits or passes the auxiliary barrier at $M_{c-c^{\alpha}}$, before the original 
barrier. The difference in curvature vanishes almost surely, so that, according to Lemma 9, $c^{-1}\left(N_{c}-c t\right)^{2}$ can be exchanged for $c^{-1}\left(M_{c-c^{\alpha}}-\left(c-c^{\alpha}\right) t\right)^{2}$. Furthermore, the difference between $N_{c}$ and $N_{l}$ equals 0 in probability as $c \rightarrow \infty$, by Lemma 10. Hence,

$$
\begin{aligned}
\lim _{c \rightarrow \infty} & \left(S_{N_{c}}-c b\left(\frac{N_{c}}{c}\right)\right) \\
& \stackrel{\mathrm{P}_{\theta}}{\rightarrow} \lim _{c \rightarrow \infty}\left(S_{N_{l}}-c l\left(\frac{N_{l}}{c}\right)-\frac{b^{\prime \prime}(t)}{2 c}\left(M_{c-c^{\alpha}}-\left(c-c^{\alpha}\right) t\right)^{2}+\frac{b^{\prime \prime}(t)}{2 c^{\alpha}}\right) .
\end{aligned}
$$

The quadratic term on the right-hand side is asymptotically a $\chi^{2}$-distributed random variable. Therefore, we have

$$
\begin{array}{r}
\lim _{c \rightarrow \infty}\left(S_{N_{l}}-c b(t)-b^{\prime}(t)\left(N_{l}-c t\right)-\frac{b^{\prime \prime}(t)}{2 c}\left(M_{c-c^{\alpha}}-\left(c-c^{\alpha}\right) t\right)^{2}\right) \\
\stackrel{\mathrm{D}_{\theta}}{\rightarrow} \lim _{c \rightarrow \infty}\left(S_{M_{c}}-c l\left(\frac{M_{c}}{c}\right)\right),
\end{array}
$$

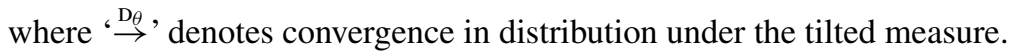

\subsection{Asymptotic independence}

Theorem 4. Under the tilted distribution, with $g^{\prime}(\theta)=b(t) / t$ and $t \leq T^{*}$, the overshoot $Z$ and $Y_{c}=a^{-1} c^{-1 / 2}\left(N_{c}-c t\right)$ are asymptotically independent, where $a^{2}=g^{\prime \prime}(\theta) t^{3}\left(b(t)-b^{\prime}(t) t\right)^{-2}$ (the variance). Therefore,

$$
\lim _{c \rightarrow \infty} \mathrm{P}_{\theta}(Z \leq z, Y \leq y)=Q_{\theta}(z) \Phi(y) .
$$

When $t=T, a^{2}=g^{\prime \prime}(\theta) \theta^{2} T^{3} / R(T)^{2}$.

Proof. Let us write $Y_{\alpha}=a^{-1} c^{-1 / 2}\left(M_{c-c^{\alpha}}-\left(c-c^{\alpha}\right) t\right)$, where $0<\alpha<\frac{1}{2}$. Furthermore, define the set

$$
A=\left\{\omega:\left|Y_{\alpha}-Y\right|<\varepsilon\right\}=\left\{\omega:\left|\Delta N-c^{\alpha} t\right|<c^{1 / 2} \varepsilon\right\} .
$$

By the law of total probability, it is possible to use this set to derive the upper bound

$$
\mathrm{P}_{\theta}(Z \leq z, Y \leq y) \leq \mathrm{P}_{\theta}\left(Z \leq z, Y_{\alpha} \leq y+\varepsilon, A\right)+\mathrm{P}\left(A^{\mathrm{c}}\right) .
$$

Remember that $\lim _{c \rightarrow \infty} \mathrm{P}_{\theta}\left(A^{\mathrm{c}}\right)=0$, by Lemma 8 .

Now, rewrite the overshoot as the overshoot of the remaining distance, i.e.

$$
Z=\Delta S_{N_{c}}-c^{\alpha} l\left(\frac{\Delta N}{c^{\alpha}}\right)+\frac{b^{\prime \prime}(t)}{2 c}\left(\Delta N+M_{c-c^{\alpha}}-c t\right)^{2}-Z_{\alpha}+O\left(\frac{\left(N_{c}-c t\right)^{3}}{c^{2}}\right) .
$$

The remainder term converges to 0 when the scale parameter goes to infinity, by Lemma 4 . Therefore, $Y_{\alpha}$ and $Z$ are asymptotically independent, conditioned on $M_{c-c^{\alpha}}$ and $S_{M_{c-c^{\alpha}}}$. Using this, Bayes's theorem, and the bounded convergence theorem, we find that

$$
\begin{aligned}
\lim _{c \rightarrow \infty} \mathrm{P}_{\theta} & \left(Z \leq z, Y_{\alpha} \leq y, A\right) \\
= & \lim _{c \rightarrow \infty} \int_{A, Y_{\alpha} \leq y+\varepsilon} \mathrm{P}_{\theta}\left(Z \leq z \mid M_{c-c^{\alpha}}, S_{M_{c-c^{\alpha}}}\right) \mathrm{d} \mathrm{P}_{\theta}\left(M_{c-c^{\alpha}}, S_{M_{c-c^{\alpha}}}\right) \\
= & \int_{Y \leq y+\varepsilon} \lim _{c \rightarrow \infty} \mathrm{P}_{\theta}(Z \leq z) \mathrm{d} \mathrm{P}_{\theta}\left(M_{c-c^{\alpha}}, S_{M_{c-c^{\alpha}}}\right) .
\end{aligned}
$$


Finally, if we apply Theorems 2 and 3, we find that

$$
\lim _{c \rightarrow \infty} \mathrm{P}_{\theta}(Z \leq z, Y \leq y) \leq Q_{\theta}(z) \Phi(y+\varepsilon) .
$$

A lower bound $Q_{\theta}(z) \Phi(y-\varepsilon) \leq \lim _{c \rightarrow \infty} \mathrm{P}_{\theta}(Z \leq z, Y \leq y)$ is found in a similar way. Thus,

$$
Q_{\theta}(z) \Phi(y-\varepsilon) \leq \lim _{c \rightarrow \infty} \mathrm{P}_{\theta}(Z \leq z, Y \leq y) \leq Q_{\theta}(z) \Phi(y+\varepsilon),
$$

but $\varepsilon$ is arbitrary and, so, $\lim _{c \rightarrow \infty} \mathrm{P}_{\theta}(Z \leq z, Y \leq y)=Q_{\theta}(z) \Phi(y)$.

Note that for arbitrary $0<\eta \neq \frac{1}{2}$ it is easy to show that $c^{-\eta}\left(N_{c}-c t\right)$ and the overshoot are asymptotically independent.

\section{Main result}

We will start by giving a heuristic large-deviation argument. The expansion of the rate function is $R(s)=R(T)+\frac{1}{2} R^{\prime \prime}(T)(s-T)^{2}$, since $T$ is determined by the equation $R^{\prime}(T)=0$. Therefore,

$$
\mathrm{P}\left(N_{c} \approx c T+c^{1 / 2} y\right) \approx \text { const } \cdot \exp \left(-c R(T)-\frac{1}{2} R^{\prime \prime}(T) y^{2}\right),
$$

where $R^{\prime \prime}(T)=R(T)^{2} / g^{\prime \prime}(\theta) \theta^{2} T^{3}+\theta b^{\prime \prime}(T)$, according to (9). The quadratic part is almost the density of a normal distribution and, therefore, one would expect that

$$
\mathrm{P}\left(N_{c} \leq c T+c^{1 / 2} y\right) \approx \text { const } \cdot \mathrm{e}^{-c R(T)} \Phi\left(\sqrt{R^{\prime \prime}(T)} y\right) .
$$

Theorem 5. Assume that the barrier $b(s)$ is convex and three-times continuously differentiable, that it satisfies $b(0)>0$, and that $\mu s<b(s)$ for all $s$. The rate of the stopping time $N_{c}$ is $R(T)=\theta b^{\prime}(T)-g(\theta) T$, where the parameters are solutions to

$$
g^{\prime}(\theta)=\frac{b(T)}{T} \text { and } g(\theta)=\theta b^{\prime}(T),
$$

and the asymptotic distribution of the first passage time is

$$
\lim _{c \rightarrow \infty} \mathrm{e}^{c R(T)} \mathrm{P}\left(N_{c} \leq c T+c^{1 / 2} y\right)=a^{-1} \eta \mathrm{E}_{\theta}\left[\mathrm{e}^{-\theta Z_{l}}\right] \Phi(y / \eta),
$$

where $a^{2}=g^{\prime \prime}(\theta) \theta^{2} T^{3} / R(T)^{2}$, the variance $\eta^{2}$ is determined by $\eta^{-2}=a^{-2}+b^{\prime \prime}(T) \theta=$ $R^{\prime \prime}(T)$, and $Z_{l}$ is the overshoot over the barrier $c b(T)+b^{\prime}(T)(n-c T)$.

In the proof of this theorem, we will tilt the probability of the stopping time as follows:

$$
\mathrm{P}_{\theta}\left(N_{c} \leq c t\right)=\mathrm{E}\left[\mathbf{1}_{\{N \leq c t\}} \sum_{n=1}^{\lfloor c t\rfloor} \exp \left(\theta S_{n}-n g(\theta)\right) \mathbf{1}_{\{N=n\}}\right]=\mathrm{E}\left[\mathbf{1}_{\{N \leq c t\}} \exp \left(\theta S_{N}-N g(\theta)\right)\right],
$$

where $\mathbf{1}_{\{\cdot\}}$ is an indicator function. The presence of the indicator function $\mathbf{1}_{\{N \leq c t\}}$ ensures that this way of changing the measure is valid even in the case in which $\mathrm{P}_{\theta}\left(N_{c}=\infty\right)>0$, with $c$ fixed.

Proof of Theorem 5. The first idea in this proof is to split the probability into two parts. Fix $\varepsilon>0$ and let $A=\left\{\left|N_{c}-c T\right|<c^{3 / 4} \varepsilon\right\}$. Then the upper large-deviation bound and the Taylor expansion of the rate function of the stopping time around $T$ give

$$
\mathrm{e}^{c R(T)} \mathrm{P}\left(N_{c} \leq c T+c^{1 / 2} y, A^{\mathrm{c}}\right) \leq 2 c T \exp \left(-\frac{1}{2} c^{1 / 2} R^{\prime \prime}(T) \varepsilon^{2}\right) \rightarrow 0 \quad \text { as } c \rightarrow 0 .
$$


The second part of the probability is

$$
\mathrm{e}^{c R(T)} \mathrm{P}\left(N_{c} \leq c T+c^{1 / 2} y, A\right)=\mathrm{E}\left[\mathbf{1}_{\left\{N_{c} \leq c T+c^{1 / 2} y\right\}} \mathrm{e}^{c R(T)}, A\right] .
$$

We tilt the distribution by adding and subtracting $g(\theta) N_{c}$ and $\theta S_{N_{c}}$ in the exponent, respectively, i.e.

$$
\mathrm{E}\left[\mathbf{1}_{\left\{N_{c} \leq c T+c^{1 / 2} y\right\}} \mathrm{e}^{c R(T)}, A\right]=\mathrm{E}_{\theta}\left[\mathbf{1}_{\left\{N_{c} \leq c T+c^{1 / 2} y\right\}} \exp \left(c R(T)+g(\theta) N_{c}-\theta S_{N_{c}}\right), A\right],
$$

where $g^{\prime}(\theta)=b(T) / T$. Then, using the fact that $S_{N_{c}}=c b\left(N_{c} / c\right)+Z$ and expanding the barrier to order three around $T$ give

$$
S_{N_{c}}=c b(T)+b^{\prime}(T)\left(N_{c}-c T\right)+b^{\prime \prime}(T) \frac{\left(N_{c}-c T\right)^{2}}{2 c}+b^{\prime \prime \prime}(\xi) \frac{\left(N_{c}-c T\right)^{3}}{3 ! c^{2}}+Z
$$

for a $\xi$ that is somewhere between $T$ and $N_{c} / c$. The third-order term in the expansion of the barrier is less than the product of $c^{1 / 4} \varepsilon^{3}$ and a bounded random variable. If we set $\varepsilon=c^{-1 / 11}$, for example, then the bound in (18) is still true and the remaining term in the expansion is of order $c^{-1 / 44}$.

Combining the rate $R(T)=\theta b(T)-g(\theta) T$ and the first derivative $R^{\prime}(T)=\theta b^{\prime}(T)-g(\theta)=$ 0 with the expansion above gives

$$
\begin{aligned}
\mathrm{E}_{\theta}\left[\mathbf{1}_{\left\{N_{c} \leq c T+c^{1 / 2} y\right\}} \exp \left(c R(T)+g(\theta) N_{c}-\theta S_{N_{c}}\right), A\right] \\
\quad=\mathrm{E}_{\theta}\left[\mathbf{1}_{\left\{N_{c} \leq c T+c^{1 / 2} y\right\}} \exp \left(-\theta \frac{b^{\prime \prime}(T)}{2 c}\left(N_{c}-c T\right)^{2}-\theta Z+O\left(c^{-1 / 44}\right)\right), A\right] .
\end{aligned}
$$

Now write $Y_{c}=c^{-1 / 2}\left(N_{c}-c T\right)$, which, by Theorem 2, converges in probability to a normal random variable $Y$ with variance $a^{2}=R(T)^{-2} g^{\prime \prime}(\theta) \theta^{2} T^{3}$. Theorem 4 implies that $Y$ and the overshoot $Z$ are asymptotically independent. Furthermore, the overshoot converges to the overshoot over a linear barrier $Z_{l}$, by Theorem 3 . We have, by the bounded convergence theorem,

$$
\begin{aligned}
\lim _{c \rightarrow \infty} & \mathrm{E}_{\theta}\left[\mathbf{1}_{\left\{N_{c} \leq c T+c^{1 / 2} y\right\}} \exp \left(-\theta b^{\prime \prime}(T) \frac{\left(N_{c}-c T\right)^{2}}{2 c}-\theta Z+O\left(c^{-1 / 44}\right)\right), A\right] \\
& =\mathrm{E}_{\theta}\left[\mathrm{e}^{-\theta Z_{l}}\right] \mathrm{E}_{\theta}\left[\mathbf{1}_{\left\{N_{c} \leq c T+c^{1 / 2} y\right\}} \exp \left(-\frac{1}{2} \theta b^{\prime \prime}(T) Y^{2}\right)\right] .
\end{aligned}
$$

Because of the squared, normally distributed random variable on the right-hand side, it is convenient to change variances, as follows. Let $\eta^{2}$ be the variance of a new normal distribution, where

$$
\eta^{-2}=\theta b^{\prime \prime}(T)+a^{-2}=\theta b^{\prime \prime}(T)+\frac{R(T)^{2}}{g^{\prime \prime}(\theta) \theta^{2} T^{3}}=R^{\prime \prime}(T),
$$

according to (9). Then

$$
\begin{aligned}
\mathrm{E}_{\theta}\left[\mathbf{1}_{\left\{N_{c} \leq c T+c^{1 / 2} y\right\}} \exp \left(-\frac{1}{2} \theta b^{\prime \prime}(T) Y^{2}\right)\right] & =\frac{1}{\sqrt{2 \pi} a} \int_{-\infty}^{y} \exp \left(-\frac{1}{2} \theta b^{\prime \prime}(T) u^{2}-\frac{u^{2}}{2 a^{2}}\right) \mathrm{d} u \\
& =\frac{\eta}{a} \Phi(y / \eta) .
\end{aligned}
$$


Now the theorem follows from the law of total probability, the 'sandwiching' inequalities

$$
\begin{aligned}
\mathrm{E}\left[\mathbf{1}_{\left\{N_{c} \leq c T+c^{1 / 2} y\right\}} \mathrm{e}^{c R(T)}, A\right] & \leq \mathrm{e}^{c R(T)} \mathrm{P}\left(N_{c} \leq c T+c^{1 / 2} y\right) \\
& \leq \mathrm{E}\left[\mathbf{1}_{\left\{N_{c} \leq c T+c^{1 / 2} y\right\}} \mathrm{e}^{c R(T)}, A\right]+\mathrm{P}\left(A^{\mathrm{c}}\right),
\end{aligned}
$$

and the fact that, in the limit as $c$ goes to infinity, the upper and the lower bounds are equal. This completes the proof.

Remark 7. When $t<T$, the first-order derivative is $R^{\prime}(t) \neq 0$. In this case, we start with computations that are almost identical to those in the proof of Theorem 5, and we find that

$$
\mathrm{e}^{c R(t)} \mathrm{P}\left(N_{c} \leq c t\right) \approx \mathrm{E}_{\theta}\left[\mathbf{1}_{\left\{N_{c} \leq c t\right\}} \exp \left(-R^{\prime}(t)\left(N_{c}-c t\right)-\theta \frac{b^{\prime \prime}(t)}{2 c}\left(N_{c}-c t\right)^{2}-\theta Z\right)\right],
$$

where, now, $g^{\prime}(\theta)=b(t) / t$ (cf. (19)). The overshoot still factors out by asymptotic independence, but the rest of the slowly changing function is not so easily handled. Heuristically, we write this expectation as a sum and exchange the probability for its Fourier inversion of the characteristic function. Then, the central part converges to the characteristic function of a normally distributed random variable. After some calculations, we find that

$$
\lim _{c \rightarrow \infty} c^{1 / 2} \mathrm{e}^{c R(t)} \mathrm{P}\left(N_{c} \leq c t\right)=\frac{\mathrm{E}_{\theta}\left[\mathrm{e}^{-\theta Z_{l}}\right]}{\left(1-\mathrm{e}^{R^{\prime}(t)}\right) \sqrt{2 \pi} a}, \quad a^{2}=g^{\prime \prime}(\theta) t^{3}\left(b(t)-b^{\prime}(t) t\right)^{-2} .
$$

This assertion is proved in [12].

\section{Acknowledgements}

I would like to thank Anders Martin-Löf and Allan Gut: Anders for his support and many ideas when working with this material; and Allan for his suggestions on how to improve an earlier version of the article. I am also grateful to Tom Britton for his suggestions regarding the writing and Tomas Höglund for fruitful discussions on the topic. Finally, I would like to thank an anonymous referee for many useful remarks and concrete suggestions on how to improve the writing and mathematics.

\section{References}

[1] Bucklew, J. A. (1990). Large Deviation Techniques in Decision, Simulation, and Estimation. John Wiley, New York.

[2] Chernoff, H. (1952). A measure of asymptotic efficiency for tests of a hypothesis based on the sum of observations. Ann. Math. Statist. 23, 493-507.

[3] Cramér, H. (1930). Mathematical risk. In Collected Works, ed. A. Martin-Löf, Springer, Berlin, 1994, p. 601.

[4] Dembo, A. And Zeitouni, O. (1993). Large Deviations Techniques and Applications. Jones and Bartlett, Boston, MA.

[5] Dembo, A., Deuschel, J.-D. And Duffie, D. (2004). Large portfolio losses. Finance Stoch. 8, 3-16.

[6] Durbin, J. (1992). The first-passage density of the Brownian motion process to a curved boundary. J. Appl. Prob. 29, 291-304.

[7] Ferebee, B. (1982). The tangent approximation to one-sided Brownian exit densities. Z. Wahrscheinlichkeitsth. 61, 309-326.

[8] Grandell, J. (1991). Aspects of Risk Theory. Springer, New York.

[9] Gut, A. (1974). On the moments and limit distributions of some first passage times. Ann. Prob. 2, $277-308$.

[10] Gut, A. (1988). Stopped Random Walks. Limit Theorems and Applications. Springer, New York.

[11] Gut, A. (1992). First passage times for perturbed random walks. Sequent. Anal. 11, 149-179.

[12] Hammarlid, O. (2004). A large deviation estimate of the first passage time to a convex barrier. Work in progress. 
[13] Heyde, C. C. (1967). Asymptotic renewal results for a natural generalization of classical renewal theory. J. R. Statist. Soc. B 29, 141-150.

[14] Höglund, T. (1979). A unified formulation of the central limit theorem for small and large deviations from the mean. Z. Wahrscheinlichkeitsth. 49, 105-117.

[15] Höglund, T. (1990). An asymptotic expression for the probability of ruin within finite time. Ann. Prob. 18, 378-389.

[16] Jennen, C. (1985). Second-order approximations to the density, mean and variance of Brownian first-exit times. Ann. Prob. 13, 126-144.

[17] Jennen, C. and Lerche, H. R. (1981). First exit densities of Brownian motion through one-sided moving boundaries. Z. Wahrscheinlichkeitsth. 55, 133-148.

[18] Lai, T. L. AND Siegmund, D. (1977). A nonlinear renewal theory with applications to sequential analysis. Ann. Statist. 5, 946-954.

[19] LaI, T. L. ANd Siegmund, D. (1979). A nonlinear renewal theory with applications to sequential analysis. II. Ann. Statist. 7, 60-76.

[20] LARsson-Cohn, L. (2001). Some remarks on "First passage times for perturbed random walks". Sequent. Anal. 20, 87-90.

[21] LundberG, F. (1926). Försäkringsteknisk Riskutjämning. F. Englunds boktryckeri AB, Stockholm.

[22] Martin-LöF, A. (1986). Entropy estimates for the first passage time of a random walk to a time dependent barrier. Scand. J. Statist. 13, 221-229.

[23] RÉNYI, A. (1957). On the asymptotic distribution of the sum of a random number of independent random variables. Acta Math. Hung. 8, 193-199.

[24] Roberts, G. O. and Shortland, C. F. (1995). The hazard rate tangent approximation for boundary hitting times. Ann. Appl. Prob. 5, 446-460.

[25] Rolski, T., Schmidli, H., Schmidt, V. And Teugels, J. (1999). Stochastic Processes for Insurance and Finance. John Wiley, Chichester.

[26] Segerdahl, C.-O. (1955). When does ruin occur in the collective theory of risk? Skand. Aktuarietidskr. 38, 22-36.

[27] Siegmund, D. (1982). Large deviations for boundary crossing probabilities. Ann. Prob. 10, 581-588.

[28] Siegmund, D. (1985). Sequential Analysis. Tests and Confidence Intervals. Springer, New York.

[29] Stam, A. J. (1967). Two theorems in $r$-dimensional renewal theory. Z. Wahrscheinlichkeitsth. 10, 81-86.

[30] Von Bahr, B. (1974). Ruin probabilities expressed in terms of ladder height distributions. Scand. Actuarial J., 190-204.

[31] Woodroofe, M. (1976). A renewal theorem for curved boundaries and moments of first passage times. Ann. Prob. 4, 67-80.

[32] Woodroofe, M. (1982). Nonlinear Renewal Theory in Sequential Analysis (CBMS-NSF Regional Conf. Ser. Appl. Math. 39). SIAM, Philadelphia, PA. 